\title{
Targets for accelerator-based research
}

\section{Proceedings of the 26th World Conference of the International Nuclear Target Development Society}

\author{
K. Eberhardt $\cdot$ J. Greene $\cdot$ B. Kindler $\cdot$ \\ B. Lommel • A. Stolarz
}

Received: 25 September 2013/Published online: 19 October 2013

(C) Akadémiai Kiadó, Budapest, Hungary 2013

\section{Foreword}

This special issue of the Journal of Radioanalytical and Nuclear Chemistry is dedicated to the 26th World Conference of the International Nuclear Target Development Society (INTDS 2012) held from August 19th to 24th, 2012 at the conference center Erbacher Hof in Mainz, Germany with special emphasis on targets for acceleratorbased research. The INTDS 2012 was co-organized by the Johannes Gutenberg-Universität Mainz and the GSI Helmholtzzentrum für Schwerionenforschung, Darmstadt. The 4th Target Fabrication Workshop (TFW), dedicated to targets for laser application and hosted by the Technical University Darmstadt, was held in parallel to the INTDS

K. Eberhardt $(\bowtie)$

Johannes Gutenberg-Universität Mainz, 55128 Mainz, Germany

e-mail: klaus.eberhardt@uni-mainz.de

K. Eberhardt

Helmholtz-Institut Mainz, Johannes Gutenberg-Universität

Mainz, 55099 Mainz, Germany

J. Greene

Physics Division, Argonne National Laboratory, Argonne, IL 60439, USA

B. Kindler · B. Lommel

GSI Helmholtzzentrum für Schwerionenforschung $\mathrm{GmbH}$, 64291 Darmstadt, Germany

A. Stolarz

Heavy Ion Laboratory, University of Warsaw, 02-093 Warsaw, Poland

2012 at the Erbacher Hof with common plenary sessions giving the participants of both events the opportunity of exchanging experiences and know-how. Accordingly, these proceedings contain some contributions presented at the 4th Target Fabrication Workshop.

The series of INTDS conferences dates back to 1963 when the "First Symposium on Research Materials for Nuclear Measurements" was held sponsored by the Central Bureau for Nuclear Measurements (CBNM, recently IRMM-Institute for Reference Materials and Measurements) in Belgium. Since then researchers involved in target preparation share experience and discuss related problems during biennial conferences hosted alternatively in Europe, North America and Asia (www.intds.org). It dates back to 1978 and 1988 when the 7th and the 14th INTDS conference were held at Garching, organized by Ludwig-Maximilians-Universität together with Technische Universität München and at Darmstadt, organized by GSI, respectively. Thus, it was delightful to meet again in Germany on the occasion of the 26th International INTDS conference.

More than 70 participating scientists from 15 countries (Belgium, Canada, France, Germany, India, Italy, Japan, Korea, Poland, Romania, Russia, South Africa, Switzerland, UK, USA), met in Mainz presenting over 50 scientific contributions covering the wide field of "Targets for Accelerator-bases Research". Two complementary visits to GSI and the TRIGA Mainz research reactor operated by the Johannes Gutenberg-Universität were included in the scientific program to introduce the institutions and laboratories.

The conference comprised seven different sessions titled "Classical Accelerator Targets", "Laser- \& Accelerator Based Research", "RIB Targets", "Isotope Enrichment and Medical Radioisotopes", "Stripper", 
"Target Characterization and Metrology", and "Actinide Targets". The sessions started with overview talks of experienced scientists followed by shorter contributions giving room to exciting new developments or methods, reports on improvements of established techniques, young scientists presenting their first scientific results as well as for introducing groups and laboratories newly joining the society.

The conference site, Erbacher Hof, combined accommodation, restaurant and conference room in a convenient way. It is situated right in the medieval part of Mainz which made it easy to socialize and discuss in varying groups. The Wednesday afternoon was dedicated to vine tasting in the vineyards located around the nearby small town of Essenheim. The day ended with a conference dinner taking place in a local restaurant. During the banquette the traditional INTDS Award of Recognition was presented to Scott Aaron from ORNL, Oak Ridge, USA, for his work on materials science of ceramic and cermet targets of enriched stable and radioactive isotopes, for serving on the Society's Board of Directors, and coorganising the 19th World Conference in Oak Ridge in 1998. The award was handed in absentia, and was accepted by a colleague participating in the conference.

The organisers of the INTDS 2012 like to express their gratitude to GSI, Helmholtzzentrum für Schwerionenforschung, Darmstadt and to Johannes Gutenberg-Universität, Mainz.

We would also like to express our special thanks to the local organizing committee for their efficient management at the conference. Gratitude is also due to the members of the International Scientific Committees of the INTDS 2012 and the 4th Target Fabrication Workshop. The guest editors would in addition like to thank the staff of JRNC for their support and co-operation in the production of these proceedings. We look forward to 27 th INTDS meeting, INTDS 2014, which will be hosted by RIKEN, Japan, in 2014.

\section{Guest editors}

- Klaus Eberhardt, Johannes Gutenberg-Universität, Mainz \& HI Mainz, Germany

- John Greene, Argonne National Laboratory, Argonne, USA

- Birgit Kindler, GSI Helmholtzzentrum für Schwerionenforschung, Darmstadt, Germany

- Bettina Lommel, GSI Helmholtzzentrum für Schwerionenforschung, Darmstadt, Germany

- Anna Stolarz, Heavy Ion Laboratory, University of Warsaw, Poland

\section{Scientific Advisory Committee}

- Christoph E. Düllmann, Johannes Gutenberg-Universität, Mainz, GSI, Darmstadt \& HI Mainz, Germany

- Klaus Eberhardt, Johannes Gutenberg-Universität, Mainz \& HI Mainz, Germany

- David Gilliam, NIST, Washington, USA

- Birgit Kindler, GSI, Darmstadt, Germany

- Bettina Lommel, GSI, Darmstadt, Germany

- Dannie Steski, BNL, Upton NY, USA

- Thomas Stöhlker, HI Jena, Germany

- Anna Stolarz, HIL-UW, Warsaw, Poland

- Atsushi Yoshida, Riken, Japan

\section{Local Organizing Committee}

- Klaus Eberhardt, Johannes Gutenberg-Universität, Mainz \& HI Mainz, Germany

- Birgit Kindler, GSI, Darmstadt, Germany

- Tatiana Litvinova, GSI, Darmstadt, Germany

- Bettina Lommel, GSI, Darmstadt, Germany

- Siglind Raiss, GSI, Darmstadt, Germany

- Petra Sach-Muth, Johannes Gutenberg-Universität, Mainz, Germany

- Brigitta Schausten, GSI, Darmstadt, Germany

\section{INTDS Review Committee}

- Scott Aaron, ORNL, Oak Ridge, USA

- Rose Boll, ORNL, Oak Ridge, USA

- Christoph Düllmann, Johannes Gutenberg-Universität, Mainz, GSI, Darmstadt \& HI Mainz, Germany

- Klaus Eberhardt, Johannes Gutenberg-Universität, Mainz \& HI Mainz, Germany

- Brian Egle, ORNL, Oak Ridge, USA

- Robert Eichler, PSI, Villigen, Switzerland

- Helmut Folger, GSI, Darmstadt, Germany

- David Gilliam, NIST, Washington, USA

- John Greene, ANL, Argonne, USA

- Ntombizonke Kheswa, iThemba LABS, Faure, South Africa

- Birgit Kindler, GSI, Darmstadt, Germany

- Bettina Lommel, GSI, Darmstadt, Germany

- Hans Maier, Munich, Germany

- Peter Maier-Komor, Munich, Germany

- Fréderique Pellemoine, Ganil, Caen, France

- Gabriel Schaumann, TU Darmstadt, Germany

- Bernhard Schlitt, GSI, Darmstadt, Germany

- Goedele Sibbens, IRMM, Geel, Belgium

- Christelle Stodel, Ganil, Caen, France 
- Anna Stolarz, HIL-UW, Warsaw, Poland

- Dannie Steski, BNL, Upton NY, USA

- Daniel W. Stracener, ORNL, Oak Ridge, USA

- Isao Sugai, KEK, Tsukuba, Japan

- Jerzy Szerypo, LMU, Munich, Germany

- Atsushi Yoshida, RIKEN, Japan

- Stefan Zeisler, TRIUMF, Vancouver, Canada

\section{Sponsors}

- BASF SE, Ludwigshafen, Germany

- Helmholtz Institute Jena, Germany

- Helmholtz Institute Mainz, Germany

- Linde AG, Mainz-Kostheim, Germany

- Urenco, Almelo, The Netherlands

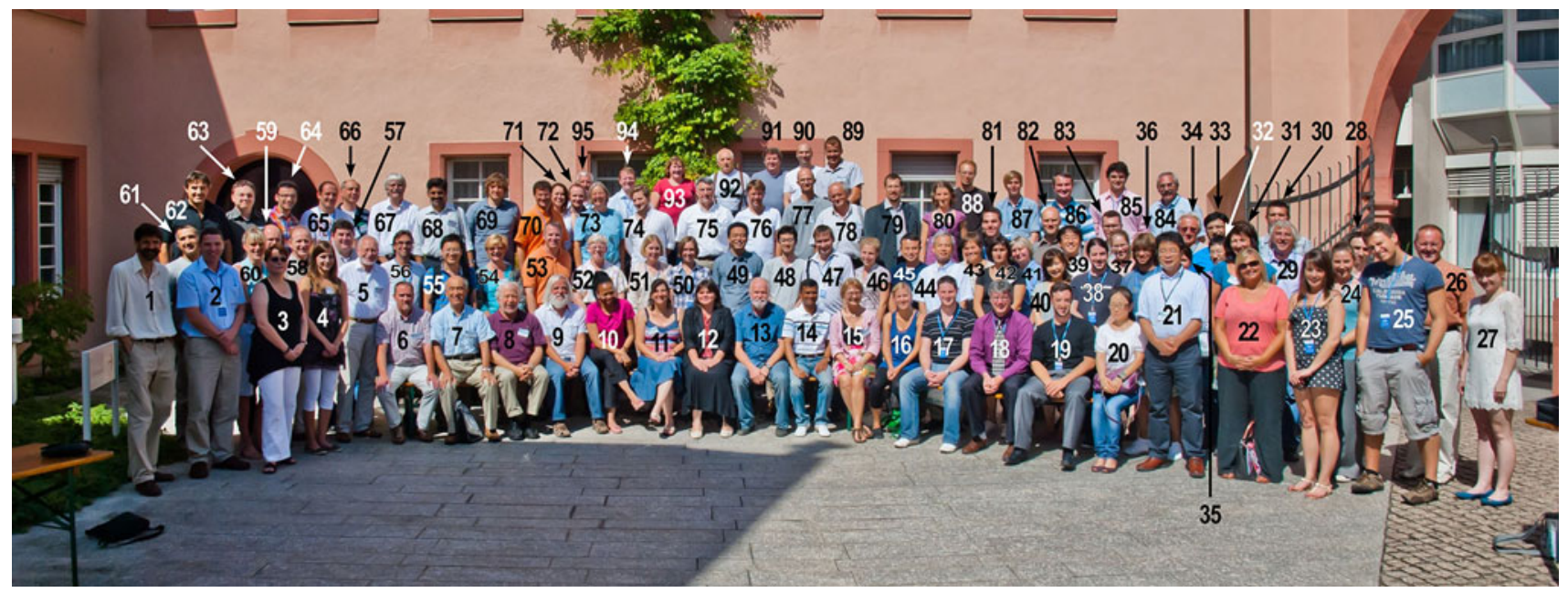




\section{Conference photo-names}

1 Martin Tolley

2 Richard Leach

3 Brigitta Schausten

4 Sarah Hebel

5 Jens Volker Kratz

6 André Moens

7 Isao Sugai

8 David Gilliam

9 Roger Eykens

10 Ntombizonke Kheswa

11 Fréderique Pellemoine

12 Marilena Tomut

13 Andrzej Lipski

14 Pranab Kumar Saha

15 Anna Stolarz

16 Katharina Kupka

17 Frank Hall

18 Nicholas Bazin

19 Paul Fitzsimmons

20 Kozue Okuno

21 Hiroki Okuno

22 Donna Wyatt

23 Michelle King

24 Stephanie Tomlinson

25 David Haddock

26 Stefan Zeisler

27 Svetlana Mironchik

28 Chris Spindloe

29 Graham Arthur

30 Frank Mertins

31 Goedele Sibbens

32 Hironori Kuboki

33 Hannan Younis

34

35 Nicoleta Florea

36 Allessandro Zani

37 Alexandra Tebartz

38 Robert Jaeger

39 Masahiro Yoshimoto

40 Masayo Yoshimoto

41 Vera Yakusheva

42 Jutta Steiner

43 Bettina Lommel

44 Koichi Yoshida

45 Atsushi Yoshida

46

47 Andrey Orekhov
48 Hiroshi Imao

49 Hiroo Hasebe

50 Lidia Borisenko

51 Nataliya Borisenko

52 Rose Boll

53 Brian Egle

54 Petra Thörle-Pospiech

55 Wenjun Ma

56 Christian Kreuzer

57 Daniel Stracener

58 Charles-Olivier Bacri

59 Jörg Runke

60 Petra Sach-Muth

61 Sav Chima

62 Denis Chatain

63 Bastian Aurand

64 Masoud Behzad

65 Jan Heyse

66 Christoph Mokry

67 John Greene

68 Sthuthikkatt Reghu Abhilash

69 Alexander Waltinger

70 Gabriel Schaumann

71 Maric Kopec

72 Stephen Gooding

73 Birgit Kindler

74 Dominik Seiler

75 Klaus Eberhardt

76 Julien Mottier

77 Jerzy Szerypo

78 Leonid Sosnin

79

80 Gabriele Hofmeister

81 Stefan Bedacht

82

83 Alessio Vascon

84 Wigen Nazarov

85 Christopher Musgrave

86 Lucian Stroe

87 Christian Schäfer

88 Alan Durnez

89 Robert Eichler

90 Andreas Beusch

91 Dannie Steski

92 Anatoliy Cheltsov

93 Megan Bennett

94 Marc Brookes

95 Colin Moss 